\title{
Sociophysics Simulations II: Opinion Dynamics
}

\author{
Dietrich Stauffer \\ Institute for Theoretical Physics, Cologne University, D-50923 Köln, Euroland
}

\begin{abstract}
Individuals have opinions but can change them under the influence of others. The recent models of Sznajd (missionaries), of Deffuant et al. (negotiators), and of Krause and Hegselmann (opportunists) are reviewed here, while the voter and Ising models, Galam's majority rule and the Axelrod multicultural model were dealt with by other lecturers at this 8th Granada Seminar.
\end{abstract}

\section{Introduction}

University professors know everything and are always right; lesser people change their opinion after interactions with others, as discussed in this review as well as in the lectures of Redner, Toral, and San Miguel. Missing at this Granada seminar was the Latané model [1, 2] which is a generalized Ising model; simulations are reviewed in [3]. Here we concentrate on the models of Sznajd [4, Krause and Hegselmann [5], and Deffuant et al [6], all three of which differ drastically in their definitions but give similar results, just as many variants of the Sznajd model were shown to have similar properties in an analytical approximation [7]. For completeness we mention that the language bit-string models of part I of our review series can also be interpreted as binary Axelrod models for multi-culturality: instead of taking over elements of another language, one may also replace elements of the native culture by those of another culture. On the other hand, the Latané model was already applied to languages in [8].

In the next section we first define the three models in a unified way, and then present, section by section, selected results.

\section{The Three Models}

Each individual $i(i=1,2, \ldots N)$ has one opinion $O_{i}$ on one particular question. This opinion can be binary ( 0 or 1$)$, multivalued integer $\left(O_{i}=\right.$ 


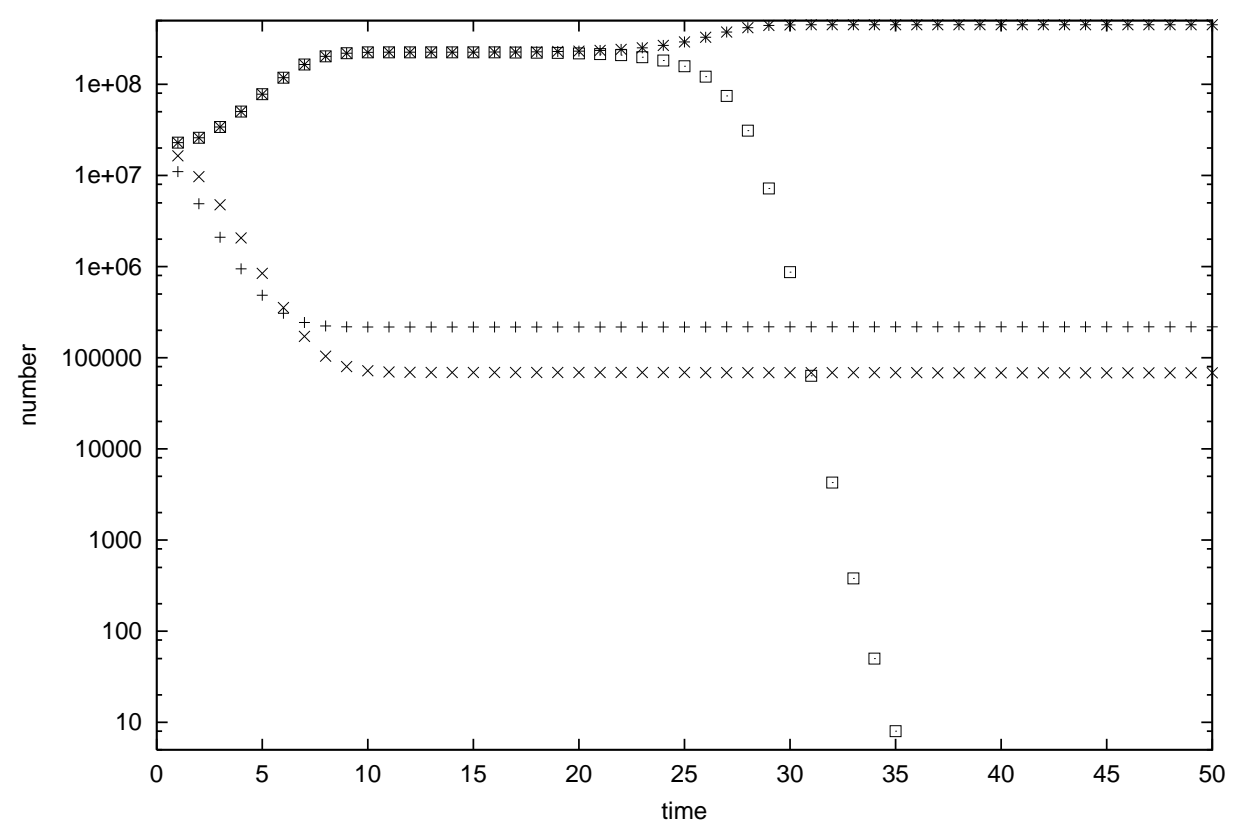

Figure 1: Continuous opinions for 450 million negotiators with everybody possibly connected to everybody: Two centrist parties fight for victory while much smaller extremist parties survive unharmed. From [9].

$1,2, \ldots, Q)$ or continuous real $\left(0 \leq O_{i} \leq 1\right)$. The neighbours $j$ of individual $i$ may be those on a square lattice, or on a Barabási-Albert network, or any other individual. Because of interactions between individuals $i$ and $j$, one of them or both may change opinion from one time step $(t)$ to the next $(t+1)$, according to rules to be specified below.

"Bounded confidence" [5, 6] means that only people with similar opinions talk to each other. If in politics five parties $1,2,3,4,5$ sit in parliament, traditionally ordered from left to right, then a left-centre coalition of 2 and 3 , or a rightist coalition of 3, 4, and 5 may work, while collaboration of the extremes 1 and 5 seldomly happen in formal coalition agreements. Thus we may assume that only parties talk to each other which differ by not more than one opinion unit, or by $\epsilon Q$ units more generally for $Q$ opinions, or by $\epsilon$ for real opinions between zero and one. If $\epsilon \geq 1$, bounded confidence is ignored; if $\epsilon \ll 1$, confidence is strongly bounded. This parameter $\epsilon$ thus 


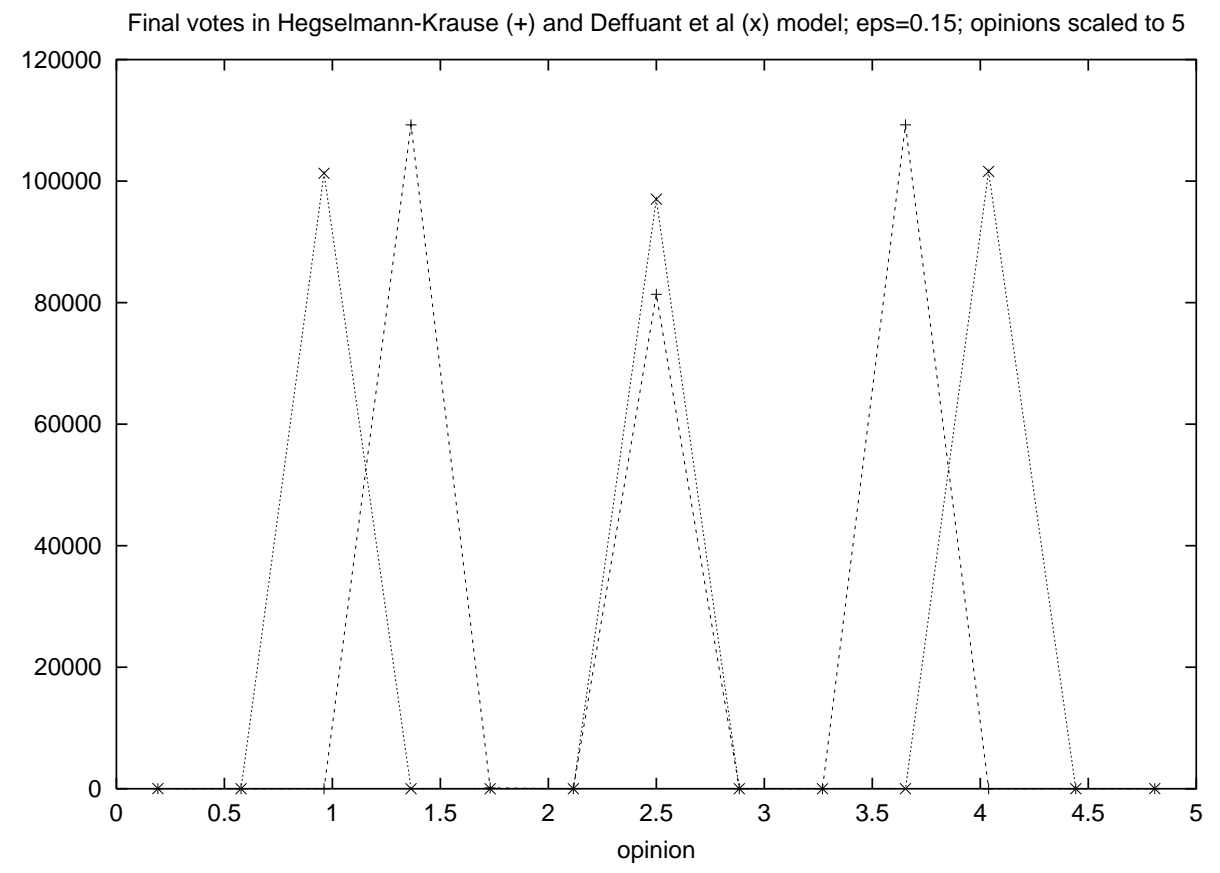

Figure 2: Final distribution of votes for 300,000 opportunists (dashed line) and negotiators (solid line), with opinions scaled from 0 to 5 instead of the original interval $[0,1]$ to facilitate comparison with missionaries, Fig.3.

measures the tolerance for dissent or the openness to different opinions.

The three models (each of which was studied in several variants) are missionaries [4], opportunists [5] and negotiators [6].

Missionaries of the Sznajd model convince all neighbours (within their confidence bound) of their mission, particularly if two neighbouring missionaries have the same opinion. For example, if on a square lattice two neighbours have the same opinion $O=2$ out of $Q=5$ possible opinions, and the confidence bound is one unit, then they force their opinion 2 onto all (at most six) lattice neighbours which before had opinions 1, 2, or 3; they cannot convince neighbours with opinions 4 or 5 .

Opportunists of the Krause-Hegselmann model ask all their neighbours (within their confidence bound) for their opinion, and then follow the arithmetic average of them. Thus for $Q=5$, a present opinion $O_{i}(t)=2$ of the considered individual and a confidence bound of one unit, the new $O_{i}(t+1)$ 


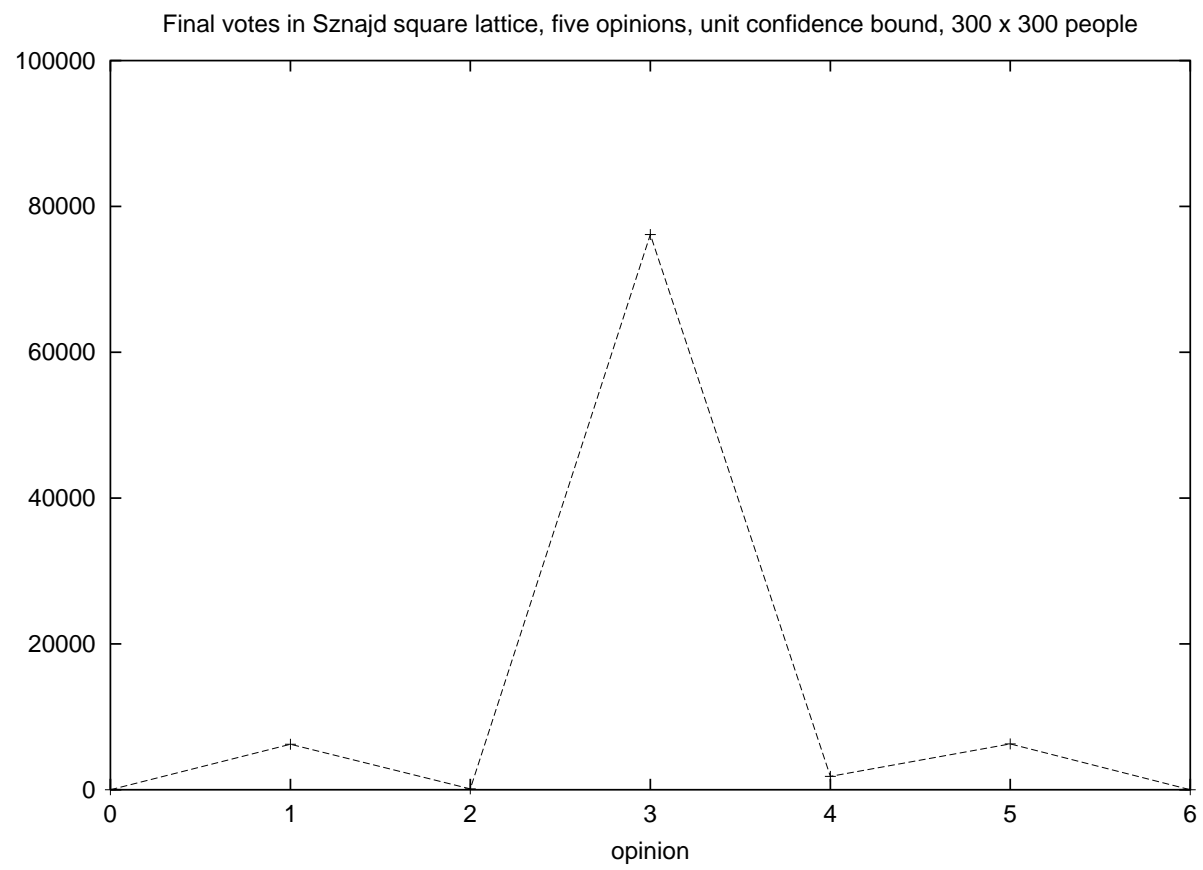

Figure 3: Final distribution of votes for $301 \times 301$ missionaries with discrete opinions 1, 2, 3, 4, 5 and unit confidence bound. Thus two neighbours of opinion 4 convince all those neighbours to the same opinion 4 which had opinions 3 or 5 before.

will be the rounded arithmetic average of all neighbour opinions except 4 and 5 .

Negotiators of Deffuant et al. each select one discussion partner at one time step. If their opinions $O_{i}$ and $O_{j}$ differ by less than the confidence bound, their two opinions mutually get closer without necessarily agreeing completely. More precisely, $O_{i}$ shifts towards $O_{j}$ and $O_{j}$ shifts towards $O_{i}$ by a (rounded) amount $\mu\left|O_{j}-O_{i}\right|$, where the extreme case $\mu=0$ means rigid unchanging opinions, while $\mu=1 / 2$ gives immediate agreement. For example, for $Q=5$ and $\mu=0.3$, for a confidence bound of three units, the pair $O_{i}=2, O_{j}=5$ will become $O_{i}=3, O_{j}=4$. (If the opinion difference is only one unit, one of the two partner takes the opinion of the other. Thus a confidence bound of only one unit makes less sense since then only this special case of one opinion jumping to the other remains, and 


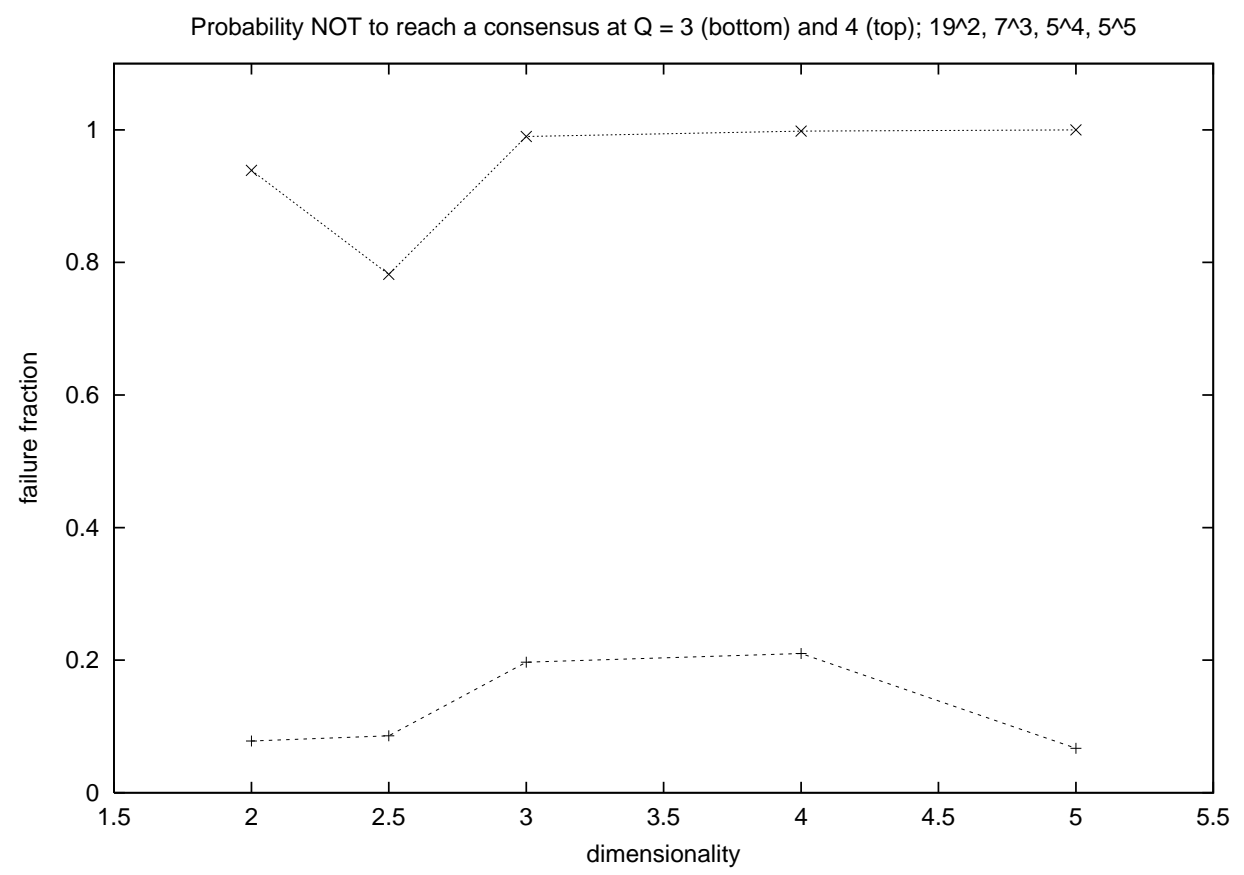

Figure 4: Probability to find no consensus with three (lower data) and four (upper data) possible missionary opinions, versus dimensionality. The triangular lattices is put at dimensionality $5 / 2$. Opinion 0 can only convince opinions $) \pm 1$; all opinions are natural numbers. From [9].

no mutual compromise as in negotiations.) Already two centuries ago, the mathematician Gauss (according to U. Krause) studied a similar problem: How do two opinions evolve if one discussion partner takes the arithmetic and the other takes the geometric average of the two opinions.

Particularly large populations can be simulated for the continuous negotiator model of infinite connection range, Fig.1. To plot the continuous opinions we binned them into 20 intervals and show only the centrist intervals 10 and 11 and the extremists in intervals $1(+)$ and $2(\mathrm{x})$. More plots on the time dependence of negotiations are given in [6].

Basic programs for missionaries, opportunists and negotiators are published in my earlier reviews [10]. 


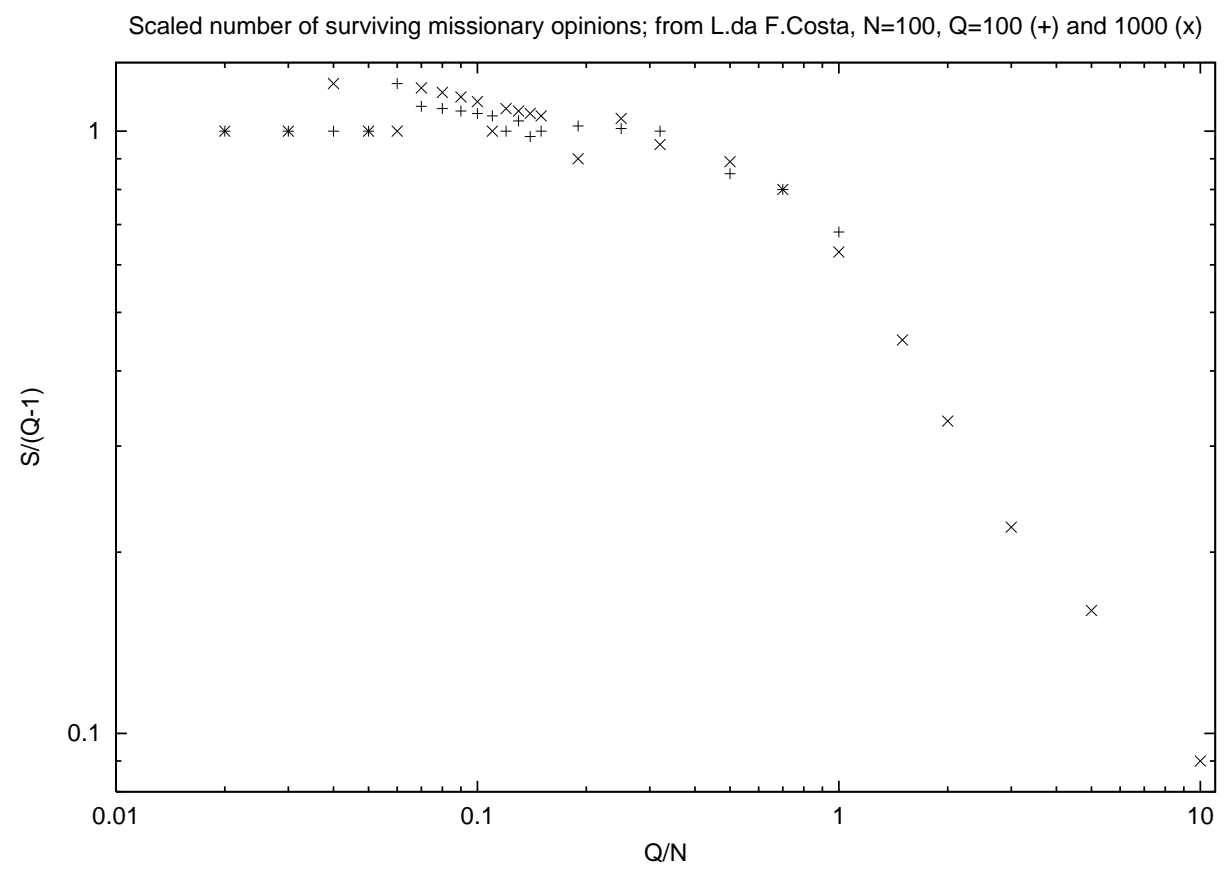

Figure 5: Scaling of the number $S$ of surviving opinions as a function of the number $Q$ of possible discrete opinions and the number $N$ of people, for missionaries. From L. da Fontura Costa, priv, comm. PRELIMINARY

\section{Consensus, Polarization or Fragmentation}

All three standard models give after sufficiently long time one of three types of results: We may find one, two or more than two different opinions surviving. The case of one opinion or consensus can also be called dictatorship. The case of two surviving opinions or polarization can also be called a balance of power between opposition and government. The case with three or more opinions or fragmentation can also be called anarchy, multi-party democracy, multiculturality or diversity. Thus the models themselves do not tell us whether the result is desirable or unwanted; this value judgement depends on the application and interpretation.

(Similarly, once we physicists have mastered the multiplication $3 \times 5=$ 15 we can estimate that three bags, of five oranges each, contain in total 15 oranges, or that a room of 5 meter length and 3 meter width has an 


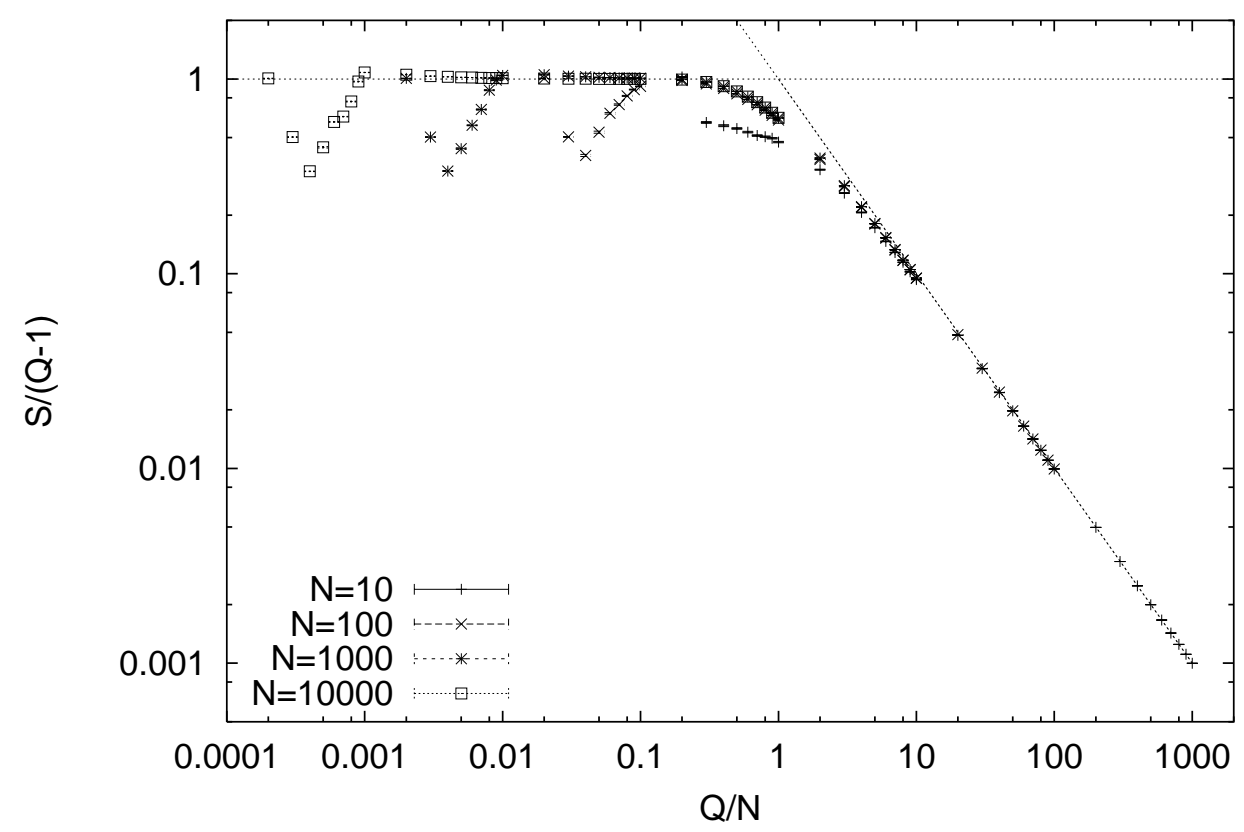

Figure 6: As Fig.5 but for opportunists. From [16].

area of 15 square meters. Both results are usually regarded as correct even though an orange does not have a surface of a square meter and a room of 15 square meters may be regarded as too large for some and too small for other purposes. Thus one model, here multiplication, may have different interpretations which can be judged differently.)

Figs. 2 and 3 show this similarity: Continuous opinions for opportunists and for negotiators give three final main opinions in Fig.2, and discrete opinions for missionaries do the same in Fig.3. The distribution of people among the three opinions may be different, with very tiny groups having opinions between the main ones, or fringe opinions near zero and one not dying out for the continuous case.

For missionaries, not only square lattices have been simulated. The original one-dimensional chains are less interesting (similar to Ising models) since they do not have a phase transition (see next section). But for triangular, simple cubic, and hypercubic in four and five dimensions the results are about the same, Fig.4: For $Q \leq 3$ possible opinions, in most cases a consen- 


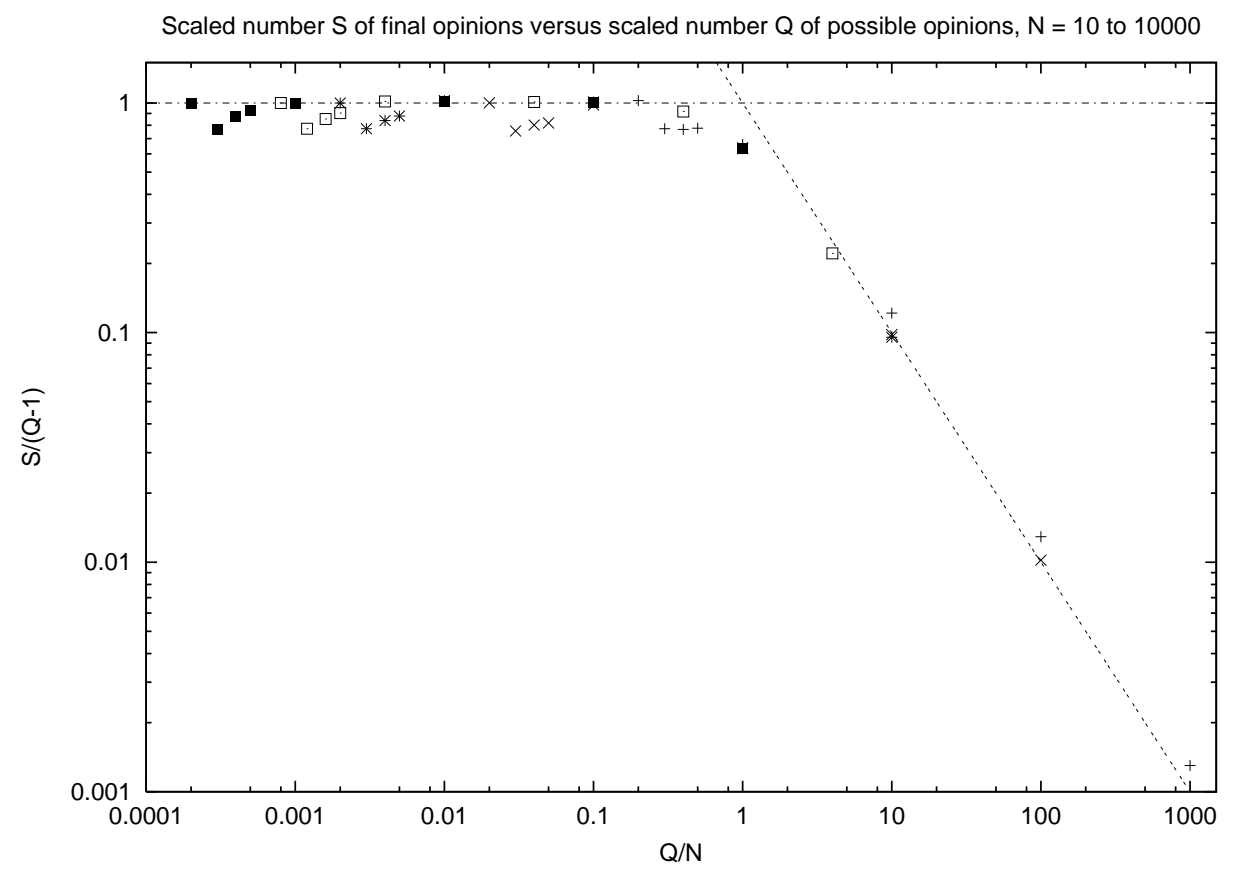

Figure 7: As Fig.5 but for negotiators, from [17].

sus is reached; for $Q \geq 4$ possible opinions, such a consensus is rare. (The confidence bound is unity for all cases.)

This threshold of 3.5 at unit confidence interval (or $\epsilon=1 / 3.5$ ) for missionaries corresponds to a threshold of $\epsilon=1 / 2$ for negotiators 11 and $\epsilon=0.2$ for opportunists [12] with continuous opinions between zero and one: For larger $\epsilon$ one has consensus, for decreasing epsilon one has first polarization into two opinions, and then fragmentation into three or more opinions, $\propto 1 / \epsilon$. The negotiator threshold $1 / 2$ is quite general [1] except if the model is made very asymmetric [13. In summary: Reaching a consensus requires a strong willingness to listen to other opinions and to reach a compromise. For negotiators the results are well described by a theory [14.

Missionaries with continuous opinions seem to reach always a consensus [15], independent of the confidence bound $\epsilon$.

Discrete instead of continuous opinions have the advantage that one can find precisely whether or not two opinions agree, without a numerical cutoff depending on the precision of the computer. Particularly opportunists could 
now be simulated in much larger numbers 16. Also, now a fixed point is reached when all opinions agree or are out of reach from each other; real numbers never fully agree and thus prevent a fixed point. Moreover, now one has a maximum number $Q$ of possible opinions, and the following scaling law is valid: If the number $N$ of people is much larger than the number $Q$ of possible opinions, then each opinion will find some followers, and the number $S$ of surviving opinions agrees with $Q$. In the opposite limit of $N \ll Q$, each person may keep its own opinion if separated by more then $\epsilon$ from the other opinions: $S=N$. It is easiest to take a unit confidence interval, i.e. $\epsilon=1 / Q$. Then for missionaries (Fig.5), opportunists (Fig.6) and negotiators (Fig.7) we get

$$
S / Q=f(Q / N)
$$

with a constant scaling function $f$ for $Q \ll N$, and $f=N / Q$ for $Q \gg N$, valid for large $Q, S, N$.

\section{Networks}

Most simulations of opportunists and negotiators had infinite connection range, i.e. each person could get into contact with all other persons, with the same probability. In contrast, the missionaries were usually simulated on lattices. Reality is in between these two extremes of nearest lattice neighbours and infinitely distant neighbours. Small world networks [18] and in particular scale-free networks of Barabási-Albert type [19] have been used as the topological basis of opinion dynamics. The name scale-free means that there is no characteristic number $k$ of neighbours for each site; instead the number of sites having $k$ neighbours decays with a power-law in $k$ like $1 / k^{3}$. These networks are supposed to describe the empirical fact than with a rather small number of steps one can connect most people in the USA with most other people there via personal acquaintances.

Their history, often misrepresented, started in 1941 with Flory's percolation theory on a Bethe lattice where each site has exactly $k$ neighbours, with the same $k$ for all sites; if the probability for two neighbours to be connected is larger than the percolation threshold $p_{c}=1 /(k-1)$, one infinite cluster of connected sites appears, coexisting with many small clusters including isolated sites. 15 years later Erdös and Rényi modified it such that each site is connected with a small probability with other sites, arbitrarily far away; this 
random graph belongs to the same "universality class" of mean-field percolation as Flory's solution but now the number $k$ of neighbours for each site fluctuates according to a Poisson distribution. We get the desired $1 / k^{3}$ law only by a very different construction (the rich get richer; or powerful people attract even more supporters). We start with $m$ sites all connected with each other. This network is then enlarged, adding one site per step. Each new site selects, among the already existing sites, exactly $m$ neighbours, randomly with a probability proportional to the number of neighbours that site had already before. Once a new site has selected an old site as a neighbour, also the new site is neighbour to the old site: this neighbourhood relation is symmetric. A computer program published in [17] at the beginning contains this construction of the network. As a result, the probability that a site has $k$ neighbours decays as $1 / k^{3}$ for $k \geq m$.

If one now wants to put opinion dynamics onto this network, one may waste much memory. With a million sites it is possible that one of them (typically one of the starting sites) has a large number of neighbours, of the order thousands. Then a neighbourhood table of size $10^{6} \times 10^{4}$ is needed. Aleksiejuk 20] programmed a one-dimensional neighbourhood table to save memory, but this is difficult to understand. It is much more practical, and does not change the results much, to switch from the above undirected networks to directed networks: The new site still selects $m$ neighbours from the old sites, but these $m$ old sites do not have the new site as a neighbour: The neighbourhood relation has become asymmetric or directed. Similarly, a new member of a political party knows the heads of that party, but these heads don't know the new member. Thus a hierarchy of directed relations is built up, which starts with the latest members of the network at the bottom and ends with the initial core members at the top.

Actually, Figs.6,7 are for a directed scale-free network, but the undirected case with infinite-range connectivity looks very similar [16] for opportunists. The simplicity of the scaling law makes it invariant against details of the network.

Also for other questions [21], negotiators with continuous opinions between zero and one on a Barabási-Albert network showed little difference between directed and undirected neighbourhoods. Fig.8 shows the resulting size distribution of opinion clusters. Such an opinion cluster is the set of people, not necessarily connected, having the same (within $10^{-6}$ ) opinion. Consensus means everybody is in one opinion cluster; thus the opposite fragmentation limit $\epsilon=0.1$ is more interesting. If we increase systematically 
the network size, we see in Fig.8 a peak moving to the right with increasing network size. This peak comes from large clusters, of the order of one per simulated network, which contain a positive fraction of the whole network. To the left of this peak, separated by a valley only for large networks, is the statistics for lots of small clusters, down to isolated people sharing their opinion with nobody else. The size distribution of these many small clusters is not much affected by the network size except that their statistics is better for larger networks. This cluster statistics is similar to percolation theory slightly above the threshold: One "infinite" cluster coexists with many finite clusters. But our clusters are sets of agreeing people, not sets of neighbouring sites as in percolation theory.

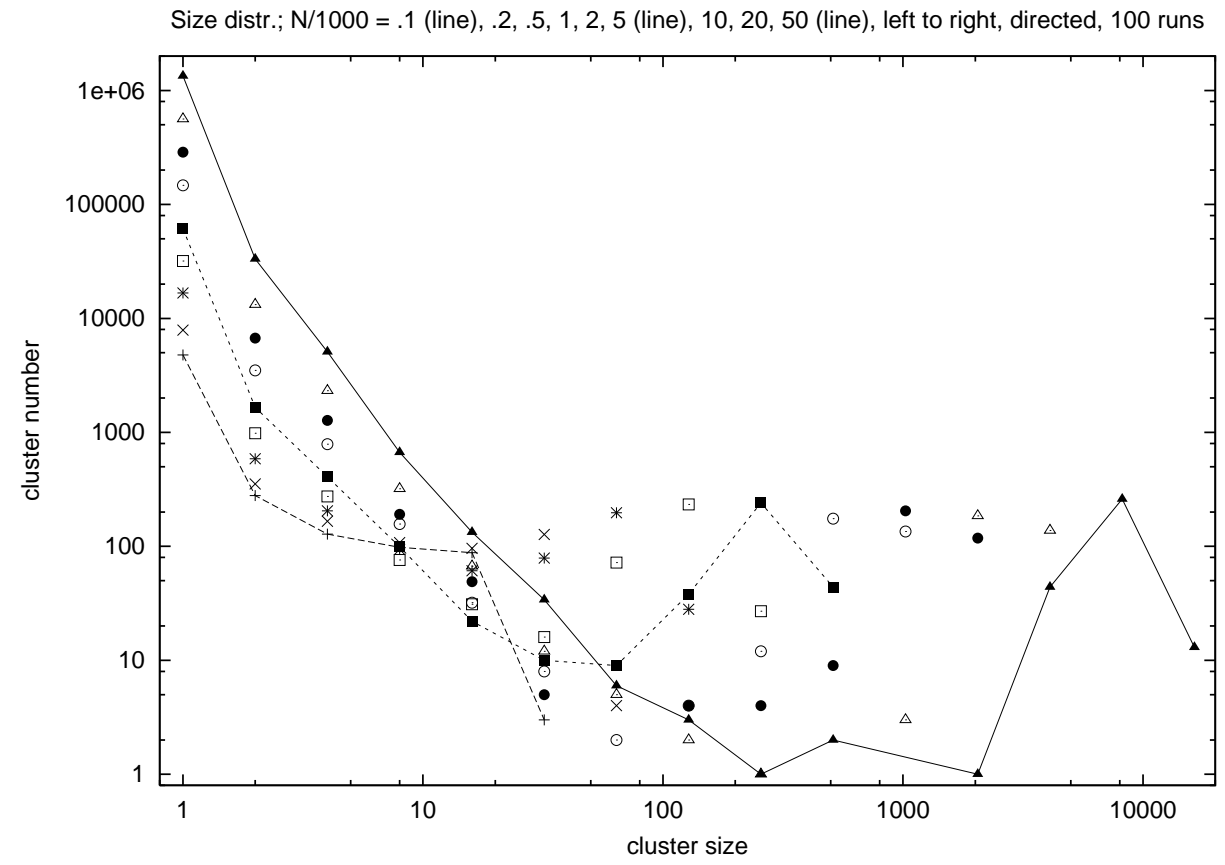

Figure 8: Size distribution of opinion clusters for negotiators on directed Barabási-Albert networks, with continuous opinions between zero and one and $\epsilon=0.1$. All network sizes, from 100 to 50,000, were simulated 100 times. From [17. 


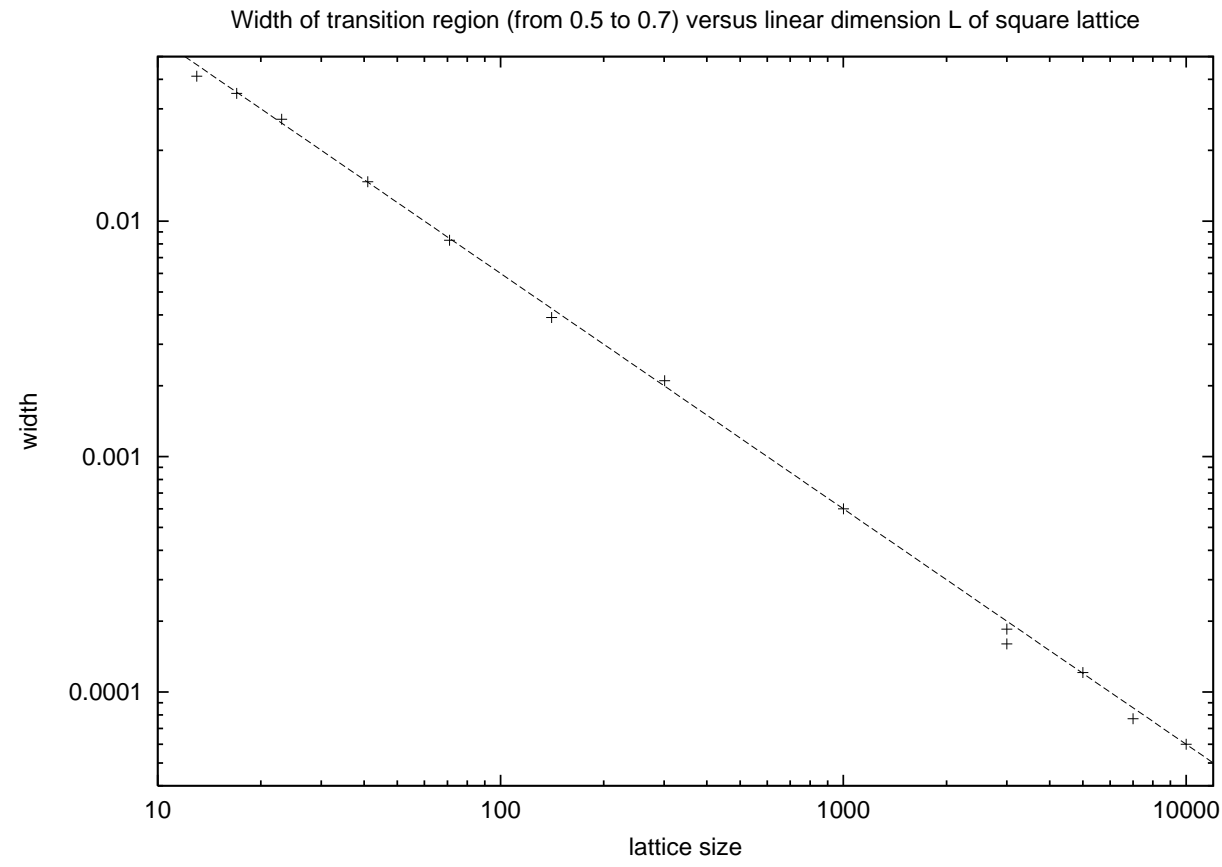

Figure 9: Transition width for missionaries with mixed global and local interaction, from [23]. This width is defined as the interval in the initial concentration of up opinions $(Q=2$ square lattice) within which the fraction of samples finishing all up increases from 50 to 70 percent.

\section{Phase Transition}

The simplest version of missionaries 4 has only $Q=2$ possible opinions up and down, in which case bounded confidence makes no sense and can be omitted. We always reach a consensus on the square lattice: Either everybody is up or everybody is down. If initially half of the opinions are up and the others are down, then after the simulation of many samples one finds 22 that half of the samples are all up and half are all down. (For negotiators, the limit $Q=2$ destroys the compromise part of shifting opinions somewhat and thus does not make sense. For opportunists we expect in this limit the same behaviour as for missionaries except that the dynamics is much faster: Each person takes the initial majority opinion.)

When we vary the initial up concentration away from 50 percent, then 
for the small and intermediate sizes for which the standard missionary model reaches its final fixed point within reasonable time, the fraction of all-up samples also moves away from 50 percent. Increasing the initial up concentration from 40 to 60 percent, we see 22 for small square lattices a slow increase of the fraction of up-samples, and for larger lattices this increase is somewhat steeper. Thus one might extrapolate that for infinite lattices one has a sharp phase transition: For initial up concentrations below 50 percent all samples finish down, and for concentrations above 50 percent all samples finish up. For the standard model the numerical evidence is meager due to small system sizes, but Schulze [23] combined the traditional nearest-neighbour interactions with global interactions similar to the nice mean-field theory of of Slanina and Laviçka [24]: two people of arbitrary distance who agree in their opinions convince their nearest neighbours of this opinion. Then, as predicted [24], the times to reach the final fixed point are much shorter and their distribution decays exponentially. Thus larger lattices can be simulated and give in Fig.8 a width of the transition varying as $1 / L$ in $L \times L$ squares, $10<L<10,000$. This is clear evidence for a simple phase transition.

In the simplification [25] suggested by a third-year student, to allow already one single missionary to convince the four square-lattice neighbours, one still has a complete consensus but no more a phase transition: The fraction of final up samples agrees with the initial concentration of up opinions, as found independently in simulation [26] and approximate theory [24; see Slanina lecture in this Seminar. Easier to understand is that in one dimension, also this phase transition does not exist [4] this absence corresponds to the lack of phase transition in the one-dimensional Ising model of 1925 which is so often mispronounced as Eyesing-Model by unhistorical speakers, instead of Eesing.

\section{Variants}

Numerous variants of the above standard models were published, and can be summarized here only shortly.

Negotiators: The inventors of negotiators [6] published several alternative, for example with unsymmetric opinion shifts as a result of compromise, with negotiators on a square lattice etc [27]. For negotiators on scale-free networks, the network was made more realistic by increasing triangular relations (the friend of my neighbours is also my own friend); the qualitative 
results remained unchanged [17]. Opinions which differ in their convincing power were simulated by Assmann [13, and the interactions of opinions on several different aspects of life by Jacobmeier [28].

Missionaries: If you dislike the complete consensus enforced by missionaries, just let their neighbours follow the convincing rule only with a high probability, or let a small minority of dissidents always be against the majority. Then the full consensus is replaced by the more realistic widespread consensus [4, 29. The role of neutrality in a three-opinion model, of opinion leaders, and of social temperature was studied by He et al [30. Sousa 31] showed that the network results are robust against the inclusion of more triangular relations (preceding paragraph) and that complete consensus can be avoided with $Q>2$. If the opinion dynamics starts already while the network is still growing not much is changed 32 .

According to [33], the missionaries are part of a wider group of cellular automata giving about the same results; see also [7. Long-range interactions, decaying with a power law of the distance on a square lattice, still need to be explained [34. Frustration occurs if we switch from sequential to simultaneous updating and one person gets different opinions simultaneously from different pairs of missionaries [35. The time-dependent decay of the number of people who never changed their opinion is Ising-like only in one dimension 36. Other (dis-)similarities with Ising models are discussed in 37, 38.

Opportunists: Fortunato [12] compared the threshold for $\epsilon$ when the number of neighbour varies proportional to the total population to the casewhere it is independent of the population size. Hegselmann and Krause [39] compared various alternative averages to the standard arithmetic average.

\section{Applications}

The most successful application of the missionary model were political elections. This does not mean that we can predict which candidate will win the next elections. Neither can statistical physics predict which air molecules will hit my nose one minute from now; the laws of Boyle-Mariott and GayLussac predict the pressure, i.e. the average number of molecules hitting my nose per picosecond. Similarly, many elections have shown a rather similar picture for the number of votes which one candidate gets (in case voters can select among numerous candidates, not among a few parties). The larger the 
number $v$ of votes is, the smaller is the number $n(v)$ of candidates getting $v$ votes. For intermediate $v$ one has $n(v) \propto 1 / v$ while for large and small $v$, downward deviations are seen: Nobody gets more than 100 percent of the votes, and nobody gets half a vote. Missionaries on their way to the consensus fixed point on a Barabási-Albert network agreed well with Brazilian votes [40, and similar agreement was also found in modified networks [41, 31] and Indian elections. However, exceptions exist (S. Fortunato, priv. comm.). It would be interesting to check whether opportunists and negotiators also agree with Brazilian election results.

If one person changes opinion, does this influence the whole community? This question, known as damage spreading in physics but invented in 1969 for genetics by Stuart Kauffman, was recently simulated in detail by Fortunato and the review [9] is still up-to-date.

Readers may try to become rich by going into advertising: How can mass media influence opinion dynamics? For missionaries [26] the answer is clear: The larger the population is the less effort is needed to convince everybody to drink Coke instead of Pepsi; but the advertizing has to come early in the opinion formation process, not when most people have already made their

choice. Again, analogous studies for opportunists and negotiators would be nice. Or perhaps you get rich with 42 .

\section{Summary}

Humans may dislike to be simulated like Ising spins, and clearly the brain is more complicated than one binary variable. But humans have been treated in this way since a long time: The astronomer Halley, known for his comet, tried to estimate human mortality already three centuries ago. Life insurance, health insurance, car insurance are present widespread examples of treating humans like inanimate particles with probabilistic behaviour, relying on the laws of large numbers. Whoever dislikes this treatment, should not blame todays sociophysicists for having started it. Already more than two millenia ago, Empedocles compared humans with fluids: Some are like wine and water, mixing well; others dislike each other, like oil and water (J. Mimkes, priv. comm.). 


\section{Summary}

I thank my collaborators working on these models since the beginning of this millenium: S. Moss de Oliveira, A.O. Sousa, J.S. Andrade, A.A. Moreira, A.T. Bernardes, U.M.S. Costa, A. Araujo, R. Ochrombel, C. Schulze, J. Bonnekoh, P.M.C de Oliveira, H. Meyer-Ortmanns, S. Fortunato, P. Assmann, and N. Klietsch.

\section{References}

[1] A. Nowak, J. Szamreij and B. Latané, Psychol. Rev. 97, 362 (1990).

[2] G.A. Kohring, J. Physique I 6, 301 (1996)

[3] J.A. Hołyst, K. Kacperski and F. Schweitzer, page 253 in: Annual Reviews of Computational Physics, edited by D. Stauffer, vol. IX, World Scientific, Singapore 2001.

[4] K. Sznajd-Weron and J. Sznajd, Int. J. Mod. Phys. C 11, 1157 (2000).

[5] R. Hegselmann and U. Krause, Journal of Artificial Societies and Social Simulation 5, issue 3, paper 2 (jasss.soc.surrey.ac.uk) (2002); U. Krause, p. 37 in: U. Krause and M. Stöckler (eds.), Modellierung und Simulation von Dynamiken mit vielen interagierenden Akteuren, Bremen University, Bremen.

[6] G. Deffuant, F. Amblard, G. Weisbuch and T. Faure, Journal of Artificial Societies and Social Simulation 5, issue 4, paper 1 (jasss.soc.surrey.ac.uk) (2002).

[7] S. Galam, preprint for Europhys. Lett. (2005).

[8] D. Nettle. Lingua 108, 95 (1999).

[9] S. Fortunato and D. Stauffer, in: Extreme Events in Nature and Society, edited by S. Albeverio, V. Jentsch and H. Kantz. Springer, Berlin Heidelberg 2005, in press.

[10] D. Stauffer, Journal of Artificial Societies and Social Simulation 5, No.1, paper 4 (2002) (jasss.soc.surrey.ac.uk). D. Stauffer: AIP Conference Proceedings 690, 147 (2 003). 
[11] S. Fortunato, Int. J. Mod. Phys. C 15, 1301 (2004).

[12] S. Fortunato, Int. J. Mod. Phys. C 16, issue 2 (2005).

[13] P. Assmann, Int. J. Mod. Phys. C 15, 1439 (2004).

[14] E. Ben-Naim, P. Krapivsky, S. Redner, Physica D 183, 190 (2003).

[15] S. Fortunato, Int. J. Mod. Phys. C 16, issue 1 (2005).

[16] S. Fortunato, Int. J. Mod. Phys. C 15, 1021 (2004).

[17] D. Stauffer, A.O. Sousa and C. Schulze, J. Artificial Societies and Social Simulation (jasss.soc.surrey.ac.uk) 7, issue 3, paper 7 .

[18] A.S. Elgazzar, Int. J. Mod. Phys. C 12, 1537 (2004).

[19] R. Albert, A.L. Barabási: Rev. Mod. Phys. 74, 47 (2002).

[20] A. Aleksiejuk, J. A. Holyst, D. Stauffer, Physica A 310, 260 (2002).

[21] D. Stauffer, H. Meyer-Ortmanns: Int. J. Mod. Phys. C 15, 241 (2004).

[22] D. Stauffer, A.O. Sousa, and S. Moss de Oliveira, Int. J. Mod. Phys. C 11,1239 (2000).

[23] C. Schulze: Int. J. Mod. Phys. C 15, 867 (2004).

[24] F. Slanina, H. Laviçka: Eur. Phys. J. B 35, 279 (2003).

[25] R. Ochrombel, Int. J. Mod. Phys. C 12, 1091 (2001).

[26] C. Schulze: Int. J. Mod. Phys. C 14, 95OC (2003) and 15, 569 (2004);

K. Sznajd-Weron and R. Weron, Physica A 324, 437 (2003).

[27] G. Deffuant et al., Adv. Compl. Syst. 3, 87 (2000) and Complexity 7, 55 (2002); G. Weisbuch, Eur. Phys. J. B 38, 339 (2004); F. Amblard and G. Deffuant, Physica A 343, 725 (2004).

[28] D. Jacobmeier, Int. J. Mod. Phys. C 16, issue 4 (2005).

[29] J.J. Schneider, Int. J. Mod. Phys. C 15, 659 (2004). 
[30] M.F. He, Q. Sun and H.S. Wang, Int. J. Mod. Phys. C 15, 767 (2004); M.F. He, ibidOB 947, M.F. He, B. Li and L.D. Luo, ibid 997.

[31] A.O. Sousa, Physica A 348, 701 (2005).

[32] J. Bonnekoh, Int. J. Mod. Phys. C 14, 1231 (2003); A.O. Sousa, Eur. Phys. J. B, in press (2005).

[33] L. Behera and F. Schweitzer, Int. J. Mod. Phys. C 14, 1331 (2003).

[34] C. Schulze, Physica A 324, 717 (20023.

[35] D. Stauffer, J. Math. Sociology 28, 25 (2004); L. Sabatelli and P. Richmond, Int. J. Mod. Phys. C 14, 1223 (2003) and Physica A 334, 274 (2004).

[36] D. Stauffer and P.M.C. de Oliveira, Eur. Phys. J. B 30, 587 (2002).

[37] K. Sznajd-Weron, Phys. Rev. E 66, 046131 (2002) and 70, 037104 (2004).

[38] A.A. Moreira, J.S. Andrade and D. Stauffer, Int. J. Mod. Phys. C 12, 39 (2001); A.T. Bernardes, U.M.S. Costa, A.D. Araujo, ibid 93.

[39] R. Hegselmann, U.Krause, http://pe.uni-bayreuth.de/?coid=18.

[40] A. T. Bernardes, D. Stauffer, J. Kertész: Eur. Phys. J. B 25, 123 (2002).

[41] M. C. Gonzalez, A. O. Sousa, H. J. Herrmann: Int. J. Mod. Phys. C 15, 45 (2004).

[42] K. Sznajd-Weron and R. Weron, Int. J. Mod. Phys. C 13, 115 (2002). 